\title{
Post-operative Complications: Can We Become More Pro-active and Less Reactive?
}

\section{Hani N Mufti $i^{1,2 *}$}

${ }^{1}$ Division of Cardiac Surgery, Department of Surgery, Queen Elizabeth II Health Sciences Centre, Dalhousie University, 2269-1796 Summer Street, Halifax, NS B3H 3A7, Canada (Current Training Institute)

${ }^{2}$ Department of Surgery, King Saud Bin-Abdulaziz University for Health Sciences, King Abdulaziz Medical City, National Guard Health Affairs, Jeddah, Saudi Arabia

\begin{abstract}
In this current era of massive datasets, and with the economic environment in its current state, funding and resources are getting scarcer. With this in mind, health care professionals should direct their focus to not only improving but also optimizing the quality of delivered care. With a shift in medicine's objectives, away from overall tallies of patients and procedures and towards patient satisfaction, quality of care and financial bottom lines, there is ever-greater pressure to find ways to maximize care in efficient ways by extracting useful information from the mountains of data being produced. Such approaches to extracting information from data are already being heavily applied in different business and marketing domains, and they are slowly finding their way into the medical domain. Data mining, in particular, is used in many industries for uncovering hidden patterns and important information that can be analyzed, summarized and presented to decision makers, executives, managers, and organizational leaders in order to help them take the appropriate decisions and actions to increase revenue, ensure customer satisfaction and guarantee prosperity. If we can imitate this business model in the medical domain, restructuring the care model from a physician-driven to a more patient-centered care. This can be accomplished by presenting the patient with the facts and information that is extracted from the similar patterns via mining of large data sets, presenting them with all possible choices, illustrating different scenarios and putting the patient in control of their own health. In doing so, we will achieve an optimal health system that is populated by well-informed and satisfied customers/patients.
\end{abstract}

Keywords: Patient centered care; Data mining; Predictive analytics; Accountable care organization

For as long as humans have existed, people have tried to analyze information in the hopes of finding patterns and making accurate predictions. A classic example is weather: in earlier times, knowing what kind of weather the coming days and weeks would bring was crucial because it affected whether people could plant, harvest, travel, and survive. Then, people had only limited information upon which to predict the weather - things like the behavior of animals and the positions of the Sun, Moon, and stars. Today, on the other hand, meteorologists use complex equations and state of the art computer systems linked to networks of satellites and weather stations around the globe, allowing for a much better understanding of weather and, as a result, more accurate predictions. These sensors gather massive amounts of information, which need to be captured, stored, normalized, and analyzed in a very short time span. However, even with the modern technology of today, there are so many variables that forecasting the weather is still not perfectly accurate.

As in meteorology, many aspects of modern life feature a growing gap between the amount of data generated and our ability to understand and make use of it. As data increases, the proportion of people who can truly understand it decreases alarmingly. Most importantly, these masses of data contain information - in many cases useful information that we do not have the capabilities and/or resources to uncover and understand [1]. However, in marketing, banking, insurance, aviation, automotive industries and many other disciplines; the massive amounts of generated data are successfully analyzed in a matter of seconds (whether by fully or partly automated processes) in order uncover useful patterns that will generate information. This information will act as the building blocks for which proper actions, applicable adjustments, and intelligent decisions will hinge on. These sectors have caught up, apparently understanding that such efficiency - and quality of the service it contributes to - keeps customers coming back and prevents waste of time and resources [1].

Several factors have contributed to the increased prominence of this approach. From a commercial point of view, the massive amount of data that needs to be collected and warehoused has always been an issue. However, computers have become cheaper and more powerful, competitive pressure is strong, and, most importantly, consumers have access to the information. If we want to take a deeper look under a scientific microscope; data collected and stored at enormous speeds, traditional techniques infeasible for raw data since it will call for physical resources that are costly (multiple human analysts and long time to drill into that massive data). Finally, from a general point of view; there is often information "hidden" in the data that are not readily evident and much of the data is never analyzed at all.

As with meteorology and the other industries already mentioned, medical science also generates incredible amounts of data. However, it's debatable whether this field makes good use of all this information - which exists primarily in the form of the knowledge synopsis within medical literature or as data points inside health records - in order to serve the ultimate goal of making and keeping people healthy. MEDLINE, U.S. National Library of Medicine's (NLM) premier bibliographic database is the most comprehensive medical literature database and contains over 19 million references to journal articles in life sciences. It includes citations from nearly 5,600 worldwide journals, and between 2,000-4,000 completed references are added each day [2]. In a study published in the Journal of Medical Library Association (JMLA)

*Corresponding author: Hani Mufti, Department of Surgery, Division of Cardiac Surgery, Queen Elizabeth II Health Sciences Centre, Dalhousie University, 2269 1796 Summer Street, Halifax, Nova Scotia B3H 3A7, Canada, Tel: +1(902) 4737597; Fax: +1 (902) 473-4448; E-mail: hani.mufti@dal.ca

Received December 23 , 2013; Accepted February 11, 2014; Published February 20, 2014

Citation: Mufti HN (2014) Post-operative Complications: Can We Become More Pro-active and Less Reactive? Surgery Curr Res 4: 178. doi:10.4172/2161 1076.1000178

Copyright: (c) 2014 Mufti HN. This is an open-access article distributed under the terms of the Creative Commons Attribution License, which permits unrestricted use, distribution, and reproduction in any medium, provided the original author and source are credited. 
in 2004, authors found that 7287 are published each month within a subset of 341 active primary care journals. A well-trained physician would need to devote about 627 hours - or 26 days! - every month so s/he can critically appraise these articles, extract useful information, and translate this information into his/her practice [3]. Of course, this is an impossibly large portion of time and, in reality, $81 \%$ of physicians report spending less than 5 hours per month reading medical journals. Clearly, there is no lack of data available; the problem is that medical professionals are drowning in data. The critical task for making use of all this data is to extract useful information and understand recurrent patterns in in an efficient manner in order to deliver evidence-based, data-supported, value-driven and patient-centered care.

There are numerous examples of authors using this non-traditional data-driven approach to tackle medical problems. There are several research articles that use such methodologies to improve early and precise detection of diseases (e.g., breast cancer, atrial fibrillation, etc.); for example, one study uses voice signal analysis for early detection of Parkinson disease [4]. Other studies use other data-driven approaches to better understand specific grouping patterns (like genetic DNA clusters, disease groups, complications patterns, infectious disease epidemics, and others). Optimization of health care resources has been also been tackled using such an approach.

The appearance of these sorts of medical publications, rooted in data-mining procedures, has increased dramatically in recent years. The first medical publication employing data mining appeared in 1978, according to PubMed [5], and was the only one that year. Their frequency increased a bit in the years that followed: there were about four such articles per year between 1982-85. Between 2010-13, though, 265 such articles have appeared each year (Figure 1).

Specific examples abound in the literature. In 2005; Yan et al. employed a clustering method called Self Organizing Map (SOM) of optic nerve images obtained by Confocal Scanning Laser Tomography (CSLT) in normal subjects and patients with glaucoma in order to devise a new five category sub-classification of glaucoma based on morphological features [6]. In another paper by Qiang et al., the researchers report a technique for recognizing vascular patterns associated with cervical lesions in colposcopic images. The lesion patterns are usually confusing and complex: even trained physicians can have difficulty recognizing typical patterns. In this paper, the authors sought to categorize images into one of six patterns. They were able to produce 24 features per image, out of which 12 were deemed relevant. Two different models were examined; one that had all 24 features and the other with only the selected 12 predictor features. By using only 12 features, the authors were able to generate a model that can differentiate each pattern with $80 \%$ accuracy. They concluded that a complex model

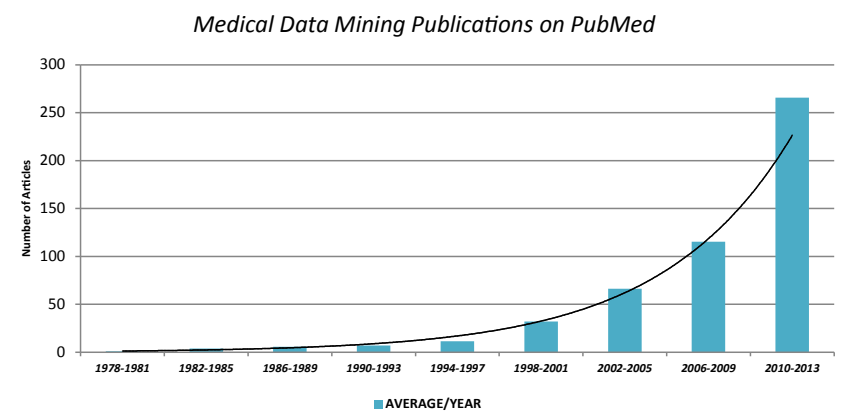

Figure 1: Medical Data Mining Publications on PubMed (Search done on the 31-January-2014) [5]. does not necessarily perform better and a simpler model can have a reasonable performance [7]. Mao et al. [8] used a comparable approach to address an important problem: early detection of deteriorating health conditions (mainly pre-shock status) in patients who are in general hospital wards (prior to transfer to intensive care). They applied a datamining framework to solve this problem, developing an Early Warning System (EWS) designed to identify the signs of clinical deterioration and provide early warning for serious clinical events. They developed this system using a very large amount of data $-41,503$ patient visits. The proposed system achieved a high specificity of $95 \%$. Further, it was able to detect deterioration in a patient health condition at least 4 hours before transfer to intensive care unit (ICU), and all the patients who were transferred to the ICU had an alert less than 24 hours before they got transferred [8].

Considering a traditional behaviour in medical care that provides a pattern of reactive care that, in my own opinion, should be transformed into a more proactive initiative is postoperative delirium. Delirium, or acute confusion, is a temporary mental disorder that occurs often among hospitalized patients and it is well established that increases hospitalization and lead to worse outcomes in general [9-14]. If we narrow our scope and focus only on a population of high risk patients that undergo complex procedures, cardiovascular surgery, and are at higher risk of developing delirium [10-12]; the reported incidence varies significantly across different studies (1-52\%) [15]. This inconsistency can be attributed to the lack of a standard definition, the absence of good detection tools and the heterogeneity of the available data. Multiple studies have shown that preventive interventions can decrease the incidence of delirium and improve outcomes $[13,14]$. Therefore, prevention and early recognition of delirium are important. Yet, conventional statistical methods have not been able to produce reliable models that can be generalized and used effectively to detect delirium.

This example provides a prime example of how the huge amounts of existing data might be harnessed and the question could be reversed and instead of asking a simple straight forward question like "How can I detect delirium?", start from scratch and collect more data; we can set out on a journey of discovery and ask the data that we possess "What can you tell me about delirium? Is there a pattern?" that will enlighten us on how should we adjust our approach. To overcome the inconsistency and heterogeneity in predicting delirium by utilizing available data mining tools to extract useful information from available data, we can improve our detection or at least be better able to flag important patients variables so that our quest will become more focused, expand on what we already have and grow closer to our goal.

Over the next few years, Accountable Care Organizations (ACOs) will come in many different forms. Yet, one underlying strategy will prove indispensable in all cases: making use of available data to drive and sustain improvement. The Centers for Medicare \& Medicaid Services (CMS) will no longer pay for preventable complications or, as they call it, "wasteful care." A 2011 report by the American Hospital Association stated that hospitals will not only need the right type of technology, but will also need to be able to extract metrics that can be used to meet the requirements of the new value-based model. Predictive analytics can be used to improve performance in two areas in which ACOs will be held accountable: reducing readmissions and providing preventive care. The basic steps in providing preventive care are identifying patients with existing conditions that may lead to more severe conditions, and acting to avert such outcomes [16].

Overall, in considering the state of data mining and information 
use in the field of medicine, one might say that we are starved for knowledge - even as we drown in data. But, of course, necessity is the mother of invention, and the use of data mining to quickly analyze massive data sets shows great potential. In this era of open access and large data sets, physicians must harness these options in order to be upto-date and acquire the best, most useful information.

To incorporate this new approach into health care, we need to attend to the foundation: medical education. It is already the case that challenges like restricted work hours for trainees are leading many training programs to shift towards competency-base models. One of these basic competencies that the new generation of physicians must acquire is the talent of utilizing the available technology to their advantage, extracting as much information as they can from the available data and combine it with existing evidence in order to make the best decisions for their patients.

In fact, with precise information extracted from the massive amounts of available data, doctors can essentially act as domain experts who present the facts and choice to the patients, who in turn can not only participate in the decision process but also have a better understanding of the situation's nuances, as well as its expectations. In this way, professionals can acknowledge patients as experts in their own health status, in control of their own decisions and actively participating in their own treatment plan. This will change not only the role of the patient but also that health care professionals, who will shift from being only the sources of knowledge and care decisions to also being advocates and promoters of optimized care based on maximum utilization of available information and resources.

Last but not least, we are about to set sail on this fascinating journey through the ocean of data and information, which will yield to amazing discoveries and astonishing knowledge. Although data mining can explain the past, the real power lies in its ability of predicting the future with great confidence. Predictive analytics will help the health care community uncover patterns and hidden relationships between data points previously buried or thought be unrelated, fill the gaps in knowledge, optimize the flow of care, and trigger -if hasn't already happened -a significant paradigm shift away from a volume-based health care system and towards a value-based health care organization.

\section{Declaration}

The Author state that this manuscript has not been published previously and is not currently being assessed for publication by any journal other than the Surgery: Current Research.

\section{Acknowledgement}

- The Author would like to acknowledge Dr. Greg Hirsch (Professor of Surgery, Dalhousie University) and Dr. Raza S. Abidi (Professor of Computer Sciences, Dalhousie University) for there continues support and advice.

- $\quad$ The Author would like to gratefully thank and acknowledge the KSAU-HS (King Saud bin-Abdulaziz University for Health Sciences) \& NGHA-SA (National Guard Health Affairs, Saudi Arabia) for the great financial and administrative support that the organization provides for its scholars and trainees.

\section{References}

1. Berman S. Capitalizing on Complexity. IBM Global Business Services, Somers, USA. 2010.

2. MEDLINE. MEDLINE Fact Sheet: U.S. National Library of Medicine; [updated 20 February 2013; cited 201319 December].

3. Brian S, Alper JAH, Susan G, Elliott, Scott Kinkade, et al. (2011) How much effort is needed to keep up with the literature relevant for primary care? Journal of Medical Library Association 92: 429-437.

4. Ramani RG, Sivagami G Parkinson Disease Classification using Data Mining Algorithms. International Journal of Computer Applications 32.

5. PubMed. Medical Data Mining: PubMed; 2014 [cited 2014 31/January/2014]

6. Yan S, Abidi SS, Artes PH (2005) Analyzing Sub-Classifications of Glaucoma via SOM Based Clustering of Optic Nerve Images. Stud Health Technol Inform 116: $483-488$

7. Ji Q, Engel J, Craine E (2000) Texture analysis for classification of cervix lesions. IEEE Trans Med Imaging 19: 1144-1149.

8. Mao Y, Chen Y, Hackmann G, Chen M, Lu C, et al. (2011) Early Deterioration Warning for Hospitalized Patients by Mining Clinical Data. International Journal of Knowledge Discovery in Bioinformatics (IJKDB) 2: 1-20.

9. Koster S, Oosterveld FG, Hensens AG, Wijma A, van der Palen J (2008) Delirium after cardiac surgery and predictive validity of a risk checklist. Ann Thorac Surg 86: 1883-1887.

10. Martin BJ, Buth KJ, Arora RC, Baskett RJ (2010) Delirium as a predictor of sepsis in post-coronary artery bypass grafting patients: a retrospective cohort study. Crit Care 14: R171.

11. Martin BJ, Buth KJ, Arora RC, Baskett RJ (2012) Delirium: a cause for concern beyond the immediate postoperative period. Ann Thorac Surg 93: 1114-1120.

12. Gottesman RF, Grega MA, Bailey MM, Pham LD, Zeger SL, et al. (2010) Delirium after coronary artery bypass graft surgery and late mortality. Ann Neurol 67: 338-344.

13. Inouye SK, Bogardus ST Jr, Charpentier PA, Leo-Summers L, Acampora D et al. (1999) A multicomponent intervention to prevent delirium in hospitalized older patients. N Engl J Med 340: 669-676.

14. Kalisvaart KJ, De Jonghe JF, Bogaards MJ, Vreeswijk R, Egberts TC, et al (2005) Haloperidol Prophylaxis for Elderly Hip-Surgery Patients at Risk for Delirium: A Randomized Placebo-Controlled Study. Journal of the American Geriatrics Society 53:1658-66.

15. Bartels K, McDonagh DL, Newman MF, Mathew JP (2013) Neurocognitive outcomes after cardiac surgery. Curr Opin Anaesthesiol 26: 91-97.

16. Bradley $P$ (2012) Predictive analytics can support the ACO model. Healthc Financ Manage 66: 102-106. 